\title{
Congenital Antithrombin Deficiency in a Pregnant Woman with Right Atrium Thrombosis
}

\author{
Feridoun Sabzi ${ }^{1}$, Donya Khosravi ${ }^{2}$, Reza Faraji ${ }^{1}$
}

ABSTRACT

BACKGROUND: One of the rare causes of venous thromboembolism in pregnancy is antithrombin III deficiency. Antithrombin III deficiency is estimated to carry a $30 \%$ risk of venous thrombotic complication during each pregnancy and postpartum.

CASE DETAILS: We present thea case of a A 21-year-old pregnant woman (Para 1+) with a history of large atrial septal defect repair at our hospital (Imam Ali Hospital, 2 May 2014). The patient, with unknown history of antithrombin III deficiency, was admitted at our emergency center with dyspnea and chest pain for the rule out of tamponade. She presented with a right atrial thrombosis in the second trimester of pregnancy despite the use of therapeutic doses of heparin and warfarin in the postoperative period as thromboembolic prophylaxis. The risk of warfarin emberyopaty led to termination of pregnancy, and successful redo-cardiac surgery outcome was achieved with the combined use of therapeutic anticoagulation and regular plasma-derived antithrombin concentrate infusions to normalize her antithrombin levels.

CONCLUSSION: She recovered from the operation uneventfully, and wad discharged in the $12^{\text {th }}$ postoperative day. In the $6^{\text {th }}$ month of follow-up, antithrombin III increased to $70 \%$ in more stable level and transethoracic echocardiography showed no recurrence of right atrial thrombus formation. This case leads to further debate regarding whether full anticoagulation should be a worthy preventive measure for venous thromboembolic prophylaxis after an open heart surgery complicated by pregnancy in a women with inherited antithrombin III deficiency. This point may become more relevant as further experience is gained with the use of recombinant human antithrombin in known cases during open cardiac surgery.

KEYWORDS: pregnancy, antithrombin u deficiency, cardiac surgery

DOI:http://dx.doi.org/10.4314/ejhs.v25i4.15

\section{INTRODUCTION}

Pregnancy is a natural thrombophilia state (TS), and the risk of thromboembolic events (TEE) in women with acquired or congenital antithrombin III deficiency (ATIIID) is increased to four or fivefold compared to non-pregnant women. If pregnancy was associated with congenital ATIIID and other acquired disturbance of coagulation factors such as cardiopulmonary bypass (CPB), this risk would increase to tenfold $(1,2)$. TEE of pregnancy occurred in $75 \%, 20 \%$ and $5 \%$ in venous, arterial and venous-arterial system respectively $(1,2)$. The true incidence of venous thromboemboli (VTE) is well known in pregnancy and could be estimated between 0.45 to 1.5 per 1000 pregnancies. The most common cause of VTE in pregnancy is a history of thrombosis that its physiopathology base was not defined with the current laboratory exam. The second most common risk factor for VTE in pregnancy is thrombophilia state (TS) $(3,4)$. TS was diagnosed in $35 \%$ of pregnant women who suffered from VTE in the pre or in the postpartum period. In this article, we present a unique case of right atrial

\footnotetext{
${ }^{1}$ Preventive Cardiovascular Research Centre Kermanshah, Kermanshah University of Medical Sciences, Kermanshah, Iran

${ }^{2}$ Department of Gynecology, School of Medicine, Shahid Beheshti University of Medical Sciences, Tehran, Iran Corresponding Author: Reza Faraji, Email: r.faraji61@gmail.com
} 
thrombus (RAT) after an atrial septal defect (ASD) repair due to congenital ATIIID in a pregnant woman. The patient was treated with fresh frozen plasma, intravenous injections of heparin and oral low-dose aspirin therapy, intravenous infusions of ATIII concentrate and surgical resection of thrombus in the right atrium. Pregnancy was terminated due to risk of warfarin emberyopathy after correction of ATIIID on the $11^{\text {th }}$ day of operation $(5,6)$. The available literature strategy for therapy of RAT in ATIIID in pregnancy is limited and the surgical approach, including various methods of the therapies, is discussed.

\section{CASE REPORT}

A 21-year-old woman, with a history of ASD repair in 5 months earlier and with unknown history of ATIIID, was admitted to our hospital at the $9^{\text {th }}$ week of gestation with dyspnea and chest pain. She did not have any familial history of thrombophilia or ATIIID, or previous VTE. TTE confirmed presence of a large thrombus in the right atrium (Figure 1). Abdominal ultrasound revealed live fetus of 8 weeks old with normal heart rate (Figure 2). Screening tests for routine coagulation test, including bleeding time (BT), prothrombin time (PT), activated partial prothrombin time (aPTT), fibrinogen and platelet function were normal. Her hepatic function tests were also normal. Blood biochemistry results were unremarkable, except for prolonged erythrocyte sedimentation rate. Thrombophilia assay except for ATIIID were normal. These normal tests include lupus anticoagulant, anticardiolipin antibodies, HLA-B5, anti-double strand DNA antibody, HLA-B27, anti-nuclear antibody, rheumatoid factor, proteins $\mathrm{C}$ and $\mathrm{S}$, antiphospholipids antibodies, factor $\mathrm{V}$ Leiden, activated protein $\mathrm{C}$ resistance, homocystein level, factors II, VII, VIII and IX. Serum level of antithrombin III was 55\% of normal range of 80 $140 \%$. The previous ASD operation was performed by $\mathrm{CPB}$ and the use of the fresh pericardial patch. To reduce further thromboemboli risk, she was managed with fresh frozen plasma and anti-thrombin concentrate and then underwent emergency surgical removal of the clot. During postcardiac surgery, fresh frozen plasma and anti-thrombin concentrate were continued and followed after termination of pregnancy. Heparin resistance was observed during previous $\mathrm{CPB}$ and in the current operation, and for maintenance of activated clotting time (ACT) level at 470 seconds, a further dose of heparin was infused to the pump prime. As to our routine postoperative strategy, intravenous heparin was started immediately following ASD surgery. Warfarin was administered orally, 12 hours after surgery to maintain the international normalized ratio (INR) at 2-2.5 level. Her previous ASD surgery was uneventful. In the $3^{\text {th }}$ month of postoperative period, with a normal cardiac TTE result, the warfarin was held and then the patient was missed from follow-up.

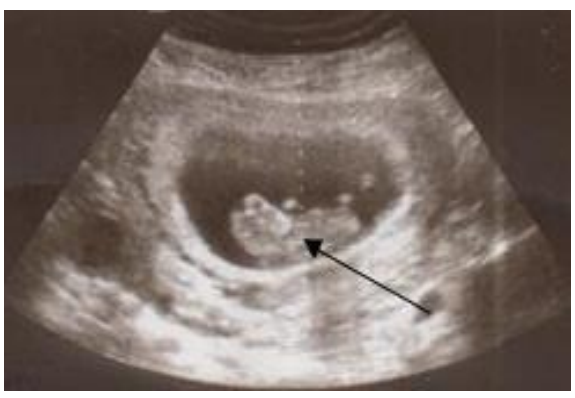

Figure 1: Abdominal sonography shows 8 weeks' live fetu (black arrow)

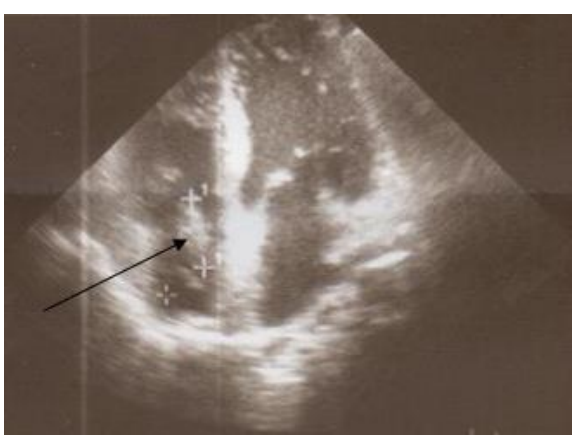

Figure 2: Shows right atrial thrombus (black arrow)

Two months later, she was admitted to our center with dyspnea and an unwished pregnancy. On physical examination, her blood pressure, pulse rate and respiratory rate were 140/70, 89 and 24 respectively. No sign of edema or thrombophelebitis was noted in lower extremities. Respiratory sound was normal in chest examination. Other clinical findings were unremarkable. The patient was admitted for evaluation of suspected VTE based on previous 
ASD repair. TTE showed a large thrombus in the right atrium with no accompanying clot in other cardiac chambers, pulmonary arteries or vena cava. Abdominal ultrasound revealed an 8 weeks old fetus with normal heart rate. Despite previous postoperative intravenous and oral anticoagulant therapy at appropriate course and dose, thrombus occurred in the right atrium although; complete thrombophilia screening tests except to ATIIID test were normal. The patient was treated with 4 units of fresh frozen plasma after receiving a loading dose and received continuous intra venous heparin. Although the patient received another large bolus dose within 6 hours, heparin infusion caused a temporary elevation in the serum aPTT level. Therefore, antithrombin concentrate was initiated with a loading dose of $50 \mathrm{iu} / \mathrm{kg}$, and the same dose was repeated at 12 hours interval, which elevated the serum AT level from 55\% to $100 \%$. The aPTT, prolonged to 76 seconds, and subsequent examination showed serum stability of the AT level compared to much more variability that had been observed prior to administration of AT concentrate therapy. The patient continued to receive IV heparin and AT concentrate for seven days. Following management of heparin resistance caused by ATD, the patient underwent a redo operation with midline sternotomy and aortic and bicava cannulation. With heparin infusion (activating clotting time of $>470$ second) and CPB establishment, ascending aorta cross clamped and cold cardioplegin infused antegradly in the aortic root. The right atrium was opened, and the large organized thrombus that was attached with a small pedicle to the lateral wall of the right atrium was removed (Figures 3,4).

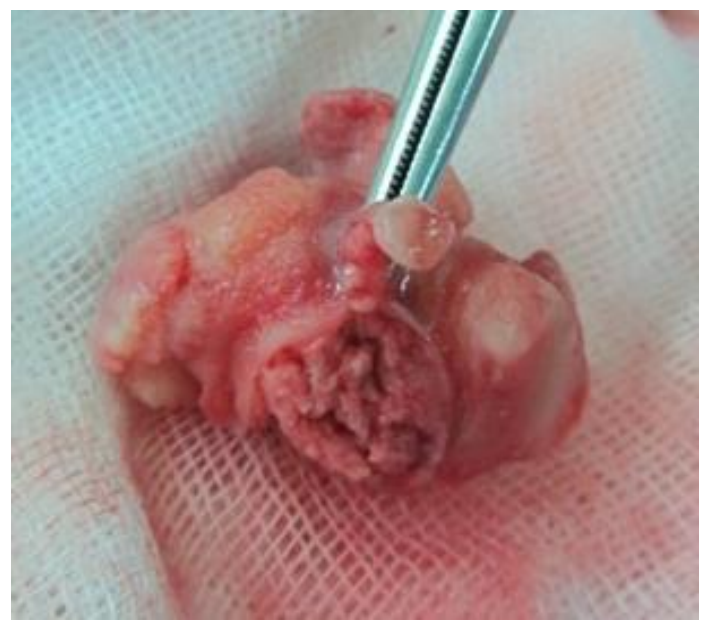

Figure 3: Shows gross pathology of resected thrombus

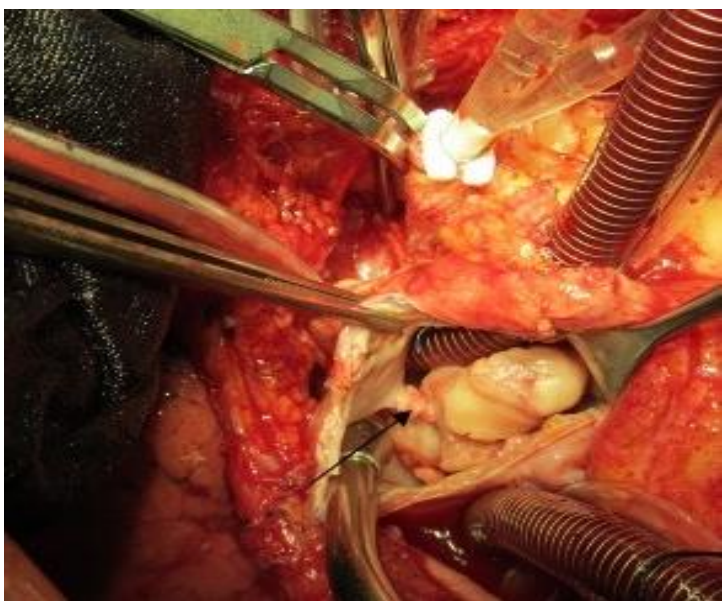

Figure 4: Shows intraoperative view of thrombus with narrow pedicle

The clot was not related to pericardial patch that was used in previous ASD closure. Pathological exam revealed fibrin and red cells in resected right atrial mass (Figure 5). At the postoperative period, AT concentrate was continued twice weekly accompanied by heparin and ASA usage. Ultimately, due to risk of warfarin emberyopathy, the pregnancy was terminated. The termination of pregnancy was associated with immediate elevation of the AT level to $65 \%$ of expected serum level. The aPTT, test examined regularly, that stabilized at 48 seconds and the AT concentrate was discontinued following termination of pregnancy. In the $6^{\text {th }}$ month of follow-up, serum level of AT reached to $70 \%$ of predicted level, but was not normalized lower limits of $80 \%$ of normal.

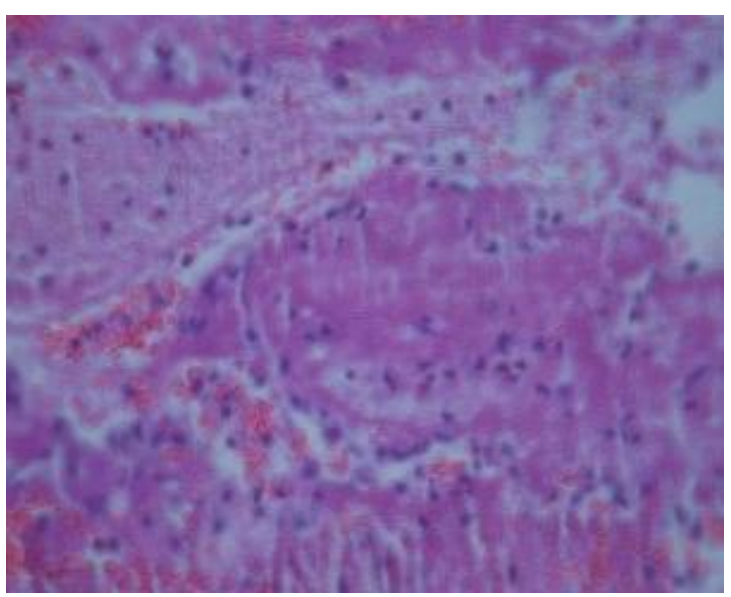


Figure 5: Shows histology of thrombus consists of red cells in fibrin background $(H \times E 40)$

\section{DISCUSSION}

RAT is a very rare event following cardiac surgery on women who had an unwished early postoperative pregnancy. Thrombophilia, behcets syndrome, myeloproliferative disorder, hepatic failure with transient antithrombin deficiency, acquired or congenital deficiency of protein $\mathrm{C}$ or ATIII and hepatocellular carcinoma are the most frequent causes of RAT $(7,8)$. ATIIID is classified into acquired and congenital types. Pregnancy is considered as an acquired type. In some pregnant women, a gradual dropping of serum level of antithrombin (AT) activity occurrs in the late trimester of pregnancy, which is not related to hypertension, preeclampsia. If combined with congenital type as in our patient, it may be associated with serious consequence. The reducuction in AT level in acquired type continues until 24 hours following delivery or termination of pregnancy. If AT serum level as a most important natural agent for the anti-coagulation of blood remains in the lower level by delaying in the delivery or termination of pregnancy, it may be associated with dangerous events like a TE in women, especially when other thrombophilia states such as cardiac surgery and CPB are added to others risk factors (9). The risk of TE increases tenfold in the presence of preeclampsia or multi-fetal pregnancy. Some, pregnant women with ATIIID may be at risk of acute fatty liver occurrence (10). Of women with ATIIID without a history of previous thrombosis, $31 \%$ will develop a TE during pregnancy. In women with a history of thrombus formation, this rate is increased to $49 \%$ (11). Cardiopulmonary bypass (CPB) may be associated with postoperative coagulophaty and enhanced thrombus formation in patients with congenital ATIIID.

Our data about RAT after open cardiac surgery with or without associated congenital ATIIID is exceedingly scanty. In this article, we presented a unique case of RAT after an ASD repair due to mild congenital ATIIID that had other predisposing risk factors in enhancing thrombophilia state of pregnancy and subsequent thrombus formation (12). These risk factors include CPB use, surgery in the right atrium with its specific geometry and relatively blood stasis, a TS of pregnancy and finally ATIIID (13). However, our data about RAT after open cardiac surgery is exceedingly scanty and our clinical experience in diagnosis and treatment of RAT formation in antithrombin deficiency in pregnancy is limited.

ATIIID has been reported with variable range of incidence in pregnancy. This variability could be related to controversy in the definition of ATD. In some case series, the thresholds of ATIIID have been considered at a higher level $(<65 \%)$ than in other series that deficiency defined at a lower level $(<50 \%)$. This challenge in the definition could be observed in related studies (14). The patient presented here was a 21-year-old pregnant woman admitted with RAT on the $8^{\text {th }}$ week of pregnancy. This clinical case report suggests that the surgeon has to appreciate that ATIIID is a very rare phenomenon, especially in pregnant women after cardiac surgery, and correct management should be started as soon as possible to avoid a serious outcome. The first inherited case of ATD was diagnosed in 1965 as a thrombophilia state, but then no case of ATIIID was reported in postcardiac surgery in pregnant woman $(15,16)$.

In conclusion, in the presence of postoperative spontaneous intracardiac thrombosis despite the use of anticoagulants in young patients, inherited antithrombin deficiency must be considered. Immobilization, intracardiac procedures, use of foreign for repair of intracardiac defect, postoperative acute or chronic infectious process increase risk of thrombosis.

\section{REFERENCES}

1. Heit JA, Kobber vig CE, James AH, Petterson TM, Bailey KR, Melton LJ 3rd. Trends in the incidence of venous thromboembolism during pregnancy or postpartum: a 30-year population-based study. Ann Intern Med 2005;143:697-706. doi:10.7326/0003-4819143-10-20051115000006.

2. Pomp ER, Lenselink AM, Rosendaal FR, Doggen CJ. Pregnancy, the postpartum period and prothrombotic defects: risk of venous thrombosis in the MEGA study. J Thromb Haemost 2008;6:632-637. doi: 10.1111/j.1538-7836.2008.02921.x.

3. Robertson L, Wu O, Langhorne P, Twaddle S, Clark P, Lowe GD, et al. Thrombophilia in 
4. pregnancy: a systematic review. $B r J$ Haematol 2006;132:171-96. doi: 10.1111/j.1365-2141.2005.05847.x

5. Vicente V, Rodriguez C, Soto I, Fernandez M, Moraleda JM. Risk of thrombosis during pregnancy and post-partum in hereditary thrombophilia. Am J Hematol 1994;46:151-2. doi: 10.1002/ajh.2830460218.

6. McColl M, Tait RC, Walker ID, Perry DJ, McCall F, Conkie JA. Low thrombosis rate seen in blood donors and their relatives with inherited deficiencies of anti- thrombin and protein $\mathrm{C}$ : correlation with type of defect, family history, and absence of the factor $\mathrm{V}$ Leiden mutation. Blood Coagul Fibrinolysis 1996;7:689-94.

7. Demers C, Ginsberg JS, Hirsh J, Henderson P, Blajch- man MA. Thrombosis in antithrombin-III-deficient persons: report of a large kindred and literature review. Ann Intern Med 1992;116:754-61. doi:10.7326/00034819-116-9-754.

8. Mateo J, Oliver A, Borrell M, Sala N, Fontcuberta J. Increased risk of venous thrombosis in carriers of natural anticoagulant deficiencies. Results of the family studies of the Spanish Multicenter Study on Thrombophilia (EMET study). Blood Coagul Fibrinalysis 1998;9:71-8.

9. Gherman RB, Goodwin TM, Leung B, Byrne JD, Hethumumi R, Montoro M. Incidence, clinical characteristics, and timing of objectively diagnosed venous thromboembolism during pregnancy. Obstet Gynecol 1999;94(5 Pt 1):730-734.

10. Peters TG, Lewis JD, Flip DJ, Morris L. Antithrombin III deficiency causing postsplenectomy mesenteric venous thrombosis coincident with thrombocytopenia. J Ann Surg 1977;9:229.
11. Michiels JJ, Stibbe J, Vellenga E, van Vliet HH. Prophylaxis of thrombosis in antithrombin III-deficient women during pregnancy and delivery. Eur J Obstet Gynecol Reprod Biol 1984;18:149-153.

12. Samson D, Stirling Y, Woolf L, Howar th D, Seghatchian MJ, de Chazal R. Management of planned pregnancy in a patient with congenital antithrombin III deficiency. $\mathrm{Br} J$ Haematol 1984;56:243-249.

13. De Stefano V, Leone G, De Carolis S, Ferrelli R, Di Donfrancesco A, Moneta E, et al. Management of pregnancy in women with antithrombin III congenital defect: report of four cases. Thromb Haemost 1988;59:193196.

14. Blondel-Hill E, Mant MJ. The pregnant antithrombin III deficient patient: management without antithrombin III concentrate. Thromb Res 1992;65:193-198.

15. Kario K, Matsuo T, Kodama K, Matsuo M. Prophylactic antithrombin III administration during pregnancy immediately reduces the thrombin hyperactivity of congenital antithrombin III deficiency by forming thrombin-antithrombin III complexes. Thromb Res 1992;66:509-515. doi:10.1016/00493848(92)90305-T.

16. Yamada T, Yamada $H$, Morikawa M, Kato EH, Kishida T, Ohnaka Y, et al. Management of pregnancy with congenital antithrombin III deficiency: two case reports and a review of the literature. $J$ Obstet Gynaecol Res 2001;27:189-197.

17. Grandone E, De Stefano V, Rossi E, Cappucci $\mathrm{F}$, Colaizzo D, Margaglione $\mathrm{M}$. Antithrombotic prophylaxis during pregnancy in women with def iciency of natural anticoagulants. Blood Coagul Fibrinolysis 2008;19:226-230. doi: 10.1097/MBC.0b013e3282f54545. 